\title{
LOS LÍMITES DE LA TESIS DE LA INCORPORACIÓN *
}

\author{
J. J. Moreso \\ Universitat Pompeu Fabra
}

RESUMEN. Este trabajo está dedicado a explorar qué condiciones deben reunir las proposiciones referidas a nuestro mundo real para poder ser combinadas con las proposiciones jurídicas y obtener nuevas proposiciones jurídicas. Las proposiciones jurídicas son el significado de oraciones prefijadas por el operador intensional «Según el derecho». El uso de oraciones con este operador produce interesantes peculiaridades de los razonamientos jurídicos: así, hay proposiciones verdaderas en nuestro mundo que no pueden combinarse con algunas proposiciones jurídicas y hay proposiciones falsas en nuestro mundo que son candidatas a integrar los razonamientos jurídicos. En este sentido, guardan alguna analogía con las proposiciones referidas a la ficción. De este modo se restringe el alcance de la —denominada por Joseph RAZ-, tesis de la incorporación, según la cual todo el derecho está basado en las fuentes o implicado por el derecho basado en las fuentes.

Palabras clave: Semántica y pragmática en el derecho, proposiciones jurídicas, razonamiento jurídico, verdad en la ficción, tesis de la incorporación.

\section{The Limits of Incorporation Thesis}

ABSTRACT. This paper deals with what conditions should have propositions referred to our actual world in order to be combined with the propositions of law and to obtain new propositions of law. The propositions of law are the meaning of sentences with the prefix «According to the law». The use of sentence with this operator reveals interesting peculiarities of legal reasoning: thus, there are true propositions in our actual world which cannot be combined with some propositions of law and there are false propositions in our present world which are candidates to figure out as premises of legal reasoning. In this sense, they show certain analogy with propositions referred to a fiction world. The so-called — by Joseph RAz - incorporation thesis, that is to say, all law is either source-based or entailed by" source-based law should be constrained for achieving plausibility.

Keywords: Semantics and pragmatics in law, propositions of law, legal reasoning, truth in fiction, incorporation thesis.

* Fecha de recepción: 12 de junio de 2015. Fecha de aceptación: 22 de septiembre de 2015.

Una versión de este trabajo fue presentada como ponencia en el Máster de Argumentación jurídica de la Universidad de Alicante, el 11 de junio de 2015. Quedo agradecido a las observaciones de todos los participantes, en especial de Josep Aguiló, Manuel AtienZA, Juan Antonio Pérez Lledó, Ángeles Ródenas y Juan RuiZ MANERO. También a las de CHIARA VALENTINI y a las múltiples conversaciones sobre la cuestión con Samuele CHILOVI. La realización de este trabajo ha sido posible gracias a la financiación del proyecto de investigación Constitucionalismo global y justicia global del Ministerio español competente y al grupo de investigación de Filosofía del Derecho de la Generalitat de Cataluña. 
Qui vult vivere, accedat, credat, incorporetur ut vivificetur.

Lectio 7, Catena Aurea in Ioannem

TOMÁs dE Aquino (2000)

\title{
1. INTRODUCCIÓN: LA ISLA DE MENORCA Y LOS GANSOS
}

\author{
todos nos parece correcto un argumento jurídico como el siguiente: \\ [1a] Según el derecho D, los compradores deben pagar el precio de la cosa \\ objeto de la compraventa. \\ [2a] Sempronia compró a Cayo un i-pad. \\ Luego
}

[3a] Según el derecho D, Sempronia debe pagar a Cayo el precio del i-pad.

Este argumento presupone algunas cosas: presupone, en primer lugar, que proposiciones como [1a] son adecuadas descripciones de lo que el derecho establece, expresan el contenido de las proposiciones jurídicas. En segundo lugar, presupone que estas proposiciones jurídicas pueden ser combinadas con otras proposiciones no-jurídicas, descripciones verdaderas del mundo, para obtener conclusiones en la forma de nuevas proposiciones jurídicas. Por tanto, al parecer las proposiciones jurídicas combinadas con las proposiciones no-jurídicas forman un conjunto clausurado mediante sus consecuencias lógicas.

Sin embargo, hay supuestos en los que este tipo de razonamientos producen resultados controvertidos. Hay casos en los que proposiciones no-jurídicas falsas, permiten concluir proposiciones jurídicas verdaderas y hay casos en los que proposiciones nojurídicas verdaderas combinadas con proposiciones jurídicas verdaderas, no permiten inferir conclusiones jurídicas verdaderas.

Veamos dos supuestos de ello. Comencemos por el caso más obvio de proposiciones falsas que son aceptables en el razonamiento jurídico. Se trata de las ficciones jurídicas ${ }^{1}$. Una famosa ficción jurídica es aquélla mediante la cual la isla de Menorca es parte de Londres. En el caso Mostyn v. Fabrigas ${ }^{2}$, Fabrigas, un residente de la isla de Menorca entonces bajo dominio inglés, fue encarcelado por Mostyn, al parecer arbitrariamente, que era el gobernador de la isla. Dado que ninguna demanda podía ser aceptada en Menorca sin el consentimiento del gobernador, Fabrigas presentó la demanda ante la Court of Common Pleas de Londres por secuestro y detención ilegal y consiguió una sentencia favorable del jurado que obligó a indemnizarle con 3.000 libras, Mostyn apeló arguyendo que el tribunal sólo tenía jurisdicción para conocer de casos entre ciudadanos residentes en Londres. No obstante, Lord Mansfield concluyó que Menorca era parte de Londres para los propósitos de la acción y el King's Bench confirmó la sentencia. Un argumento que puede ser formalizado del siguiente modo:

1 Ésta era la posición de FuLLER, 1967: 9. Matizaciones a dicha posición en HeRNÁNDEZ MARín, 1986; MENDONCA, 1998; SCHAUER, 2015.

2 Mostyn v. Fabrigas, 1 Cowp. 161, 98 Eng. Rep 1021 (K. B. 1774) (Mansfield, L. J.). Contado en muchos lugares, vid., por ejemplo, SCHAUER, 2015: 122. 
[1b] Según el derecho D, el tribunal T sólo tiene jurisdicción sobre los ciudadanos residentes en Londres.

[2b] Menorca está en Londres.

[3b] Según el derecho D, el tribunal T tiene jurisdicción sobre los ciudadanos residentes en Menorca.

Éste parece ser un buen argumento jurídico, aunque la proposición contenida en [2b] es patentemente falsa. De hecho, las ficciones jurídicas pueden contemplarse como proposiciones falsas, que el derecho toma como si fuesen verdaderas para determinados efectos. En el Código Civil español (art. 29) se establece que el concebido se tiene por nacido a los efectos que le sean favorables; aunque es obvio que los concebidos son, por definición (necesariamente) no nacidos. En el Código Penal español (art. 239) se establece que se considerarán llaves falsas las legítimas perdidas por su dueño; contra la obviedad que las llaves legítimas (otra vez de manera necesaria) no pueden ser falsas ${ }^{3}$. Las presunciones, en cambio, son proposiciones contingentemente falsas que, de forma definitiva o salvo en prueba en contrario, se toman como verdaderas: así el code Napoléon de 1804 establecía (en el art. 312): «L'enfant conçu pendant le mariage a pour père le mari». Y establecía, sólo a solicitud del marido, unas excepciones muy rígidas. Es obvio que aquí estamos ante una proposición contingentemente verdadera, es decir, que bien puede ser falsa.

Pasemos ahora a un caso en el que una proposición no-jurídica verdadera no permite obtener, cuando es combinada con determinadas proposiciones jurídicas, proposiciones jurídicas verdaderas. Se trata de una historia referida a la Edad Media. Al parecer algunos naturalistas medievales pensaron que los gansos, a los que no veían reproducirse, no eran aves sino animales que evolucionaban a partir de los percebes, por la similitud de estos mariscos con las patas de los gansos. Apoyados en esta conjetura algunos clérigos, así en Irlanda, arguyeron que los gansos eran una comida permitida por la doctrina católica (DC) los viernes de cuaresma, junto con el pescado y el marisco, y no prohibida como las carnes rojas y las carnes blancas ${ }^{4}$. Tomemos esta historia por cierta y supongamos que era saber común en algunas comunidades católicas en la Edad Media, entonces aunque es verdad que

[1c] Según la DC, los viernes de cuaresma no se puede comer carne

y es verdad que

[2c] Comer un plato cocinado con ganso es comer carne;

es falso que

[3c] Según la DC, los viernes de cuaresma no se puede comer un plato cocinado con ganso.

3 Para dar cuenta de la semántica de estas proposiciones, dado que son falsas necesariamente, necesitaríamos recurrir a la noción de mundos imposibles. No me ocuparé de ello, pero hay una literatura cada vez más relevante al respecto, vid., por ejemplo, BERTO, 2013.

4 Vid. Friedman y FigG (2000: 47). F. SCHAUER (2015: 122, nota 14) cuenta una historia semejante, pero más reciente, tal vez apócrifa, según la cual en 1939 los famosos y queridos ciervos que corretean por los parques del Magdalen College en Oxford tenían el riesgo de ser requisados durante la guerra para ser destinados a alimento por el Ministerio competente. Para evitar dicha eventualidad, se dice que algunos de los influyentes graduados del Magdalen, altos funcionarios en el gobierno, consiguieron que los ciervos fueran reclasificados como verduras y así salvados del matadero. 
[2c] no forma parte de las creencias relevantes de la comunidad, sino precisamente su negación.

Bien, pues precisamente de estos casos voy a ocuparme en este trabajo. Para dar cuenta de estas peculiaridades, será preciso analizar adecuadamente el comportamiento lógico del operador «Según el derecho» (sinónimo de «El derecho establece que», «Conforme al derecho», «Jurídicamente», etc.) $)^{5}$. Comenzaré exponiendo, con cierto detalle, la tesis de la incorporación, según la cual al derecho pertenecen no sólo las normas basadas en las fuentes sino también todo aquello que está implicado por ellas. Pasaré, a continuación, a mostrar que el operador «Según el derecho», es un operador intensional y, como todos los operadores intensionales, su comportamiento semántico y pragmático tiene peculiaridades que merecen ser destacadas. Y terminaré proponiendo un conjunto de restricciones que deben ser tomadas en cuenta en relación con el comportamiento lógico de las proposiciones jurídicas y de las proposiciones nojurídicas en los razonamientos jurídicos.

\section{LA TESIS DE LA INCORPORACIÓN}

En un trabajo de Joseph RAZ, un trabajo seminal para esta cuestión, publicado en 1985 en The Monist ${ }^{6}$, se distingue entre tres tesis que han dominado el debate desde entonces:

La tesis de las fuentes: todo el derecho está basado en las fuentes.

La tesis de la incorporación: todo el derecho está o bien basado en las fuentes o bien implicado por el derecho basado en las fuentes.

La tesis de la coherencia: todo el derecho consiste en el derecho basado en las fuentes junto con la justificación moralmente más fundada del derecho basado en las fuentes.

Este trabajo de RAZ está dedicado a argüir a favor de la tesis de las fuentes y en contra de las otras dos tesis. Ésta es su forma de vindicar el denominado positivismo jurídico excluyente, en contra del positivismo jurídico incluyente, que defiende la tesis de la incorporación y del antipositivismo dworkiniano, que defiende la tesis de la coherencia ${ }^{7}$.

Aunque considero, con las restricciones que resultan de este trabajo, la tesis de la incorporación adecuada y, ampliada convenientemente, también creo que puede hacer plausible la tesis de la coherencia; argumentar a favor de dichas posiciones me llevaría demasiado lejos de los propósitos de este trabajo. La versión raziana de la tesis de la incorporación tiene, al menos dos lecturas. Según la primera versión, la versión débil (TID) ${ }^{8}$ :

5 Una idea notada por HeRnÁndez MARÍN, 1984, y por RAZ, 1994: 197.

6 Ahora en RAZ, 1994, vid. también más recientemente RAZ, 2004.

7 Presentaciones panorámicas de estas posiciones en MARMOR, 2002; HimmA, 2002; MORESO, 2015, para el positivismo jurídico excluyente e incluyente. Para DWORKIN puede verse el propio DwORKIN, 2006: cap. 8.

8 A menudo dicha tesis se entiende como la incorporación al derecho de pautas y argumentos morales. Yo mismo la he presentado del siguiente modo (MORESO, 2015: 174):

Cuando las fuentes del derecho (la Constitución, las leyes, los precedentes judiciales, por ejemplo) incluyen conceptos y consideraciones morales, lo que el derecho establece ha de ser identificado mediante el uso de la argumentación moral. 
(TID): las normas que son consecuencia lógica de normas válidas son también válidas.

Esta tesis es el núcleo de la contribución a la teoría del derecho de aquellas concepciones que sostienen que los sistemas normativos son sistemas estructurados mediante la relación de consecuencia lógica, son conjuntos de normas clausurados mediante sus consecuencias lógicas?

La segunda versión es más fuerte que la primera, puesto que sostiene:

(TIF): las normas que son consecuencia lógica de normas válidas y de proposiciones verdaderas son también normas válidas.

Esta es la tesis que nos permite decir, por ejemplo, que si hay una norma válida que obliga a los jueces a castigar con una sanción $\mathrm{S}$ al funcionario que acepta sobornos y es verdad que el funcionario Ticio ha aceptado sobornos, entonces un juez debe castigar a Ticio a la sanción S. Para la tesis débil, en cambio, sólo podríamos concluir que si Ticio ha obtenido sobornos entonces Ticio debe ser castigado por un juez. Las conocidas objeciones de KELSEN a la aplicación de la lógica a las normas sólo se refieren a la versión fuerte, la versión débil le parece aceptable. Veamos cómo se expresa KELSEN (1991: cap. 58, 250):

Puede asumirse que el legislador que quiere que alguien que causa la muerte de otro por su conducta deba ser condenado a reclusión perpetua, también quiere que alguien que causa la muerte de otro mediante un disparo deba ser condenado a reclusión perpetua, puesto que puede asumirse que el legislador sabe que uno puede matar a otro si le dispara. Pero no puede asumirse que el legislador quiera que Schuster, quien disparó a Bauer causándole la muerte, ha de ser condenado a reclusión perpetua, puesto que no puede asumirse que el legislador sabe que Schuster disparará a Bauer causándole la muerte [...] La relación entre ambas normas es presentada en la forma de una inferencia:

$\mathrm{Si}$ alguien causa de manera intencional la muerte de otro, debe ser condenado a reclusión perpetua.

Disparar puede causar la muerte de una persona.

Luego, alguien que intencionalmente dispara a otro causándole la muerte, debe ser condenado a reclusión perpetua.

Pero esta inferencia no conlleva la validez de norma nueva alguna. La norma presentada como conclusión es ya válida si la norma representada como premisa mayor es válida, puesto que la primera está implícita en esta última.

Es decir, KELSEN no cuestiona las relaciones lógicas entre las normas, sino las relaciones lógicas entre las normas generales, las proposiciones que describen los hechos y las normas individuales. O sea KELSEN acepta la versión débil de la tesis de la incorporación, aunque rechaza la tesis fuerte.

La posición de RAZ, en cambio, no es contraria a que algunas normas implícitas sean válidas, si es posible atribuirlas a las autoridades que las promulgaron.

La presentación raziana es mucho más general y de mayor interés para lo que deseo desarrollar aquí. Al final del trabajo, sin embargo, volveré a la cuestión de la incorporación de pautas morales. Sin embargo, la caracterización raziana hace que posiciones como las referenciadas en la nota siguiente aparezcan como incorporacionistas, cuando algunos de ellos (en especial BuLYGIN, vid. BULYGIN, 2006) rechazan explicítamente la incorporación de pautas morales en el derecho, aún siendo uno de los más perspicuos defensores de la concepción según la cual las normas que son consecuencia lógica de las normas válidas son también válidas.

9 Vid., por ejemplo, vON Wright, 1963; AlCHOURRÓN-Bulygin, 1971; CARACCIOLO, 1988; RodríGUEZ, 2002; MARANHÃo, 2013; NAVARRO-RODRÍGUEZ, 2014. 
Sin embargo, rechaza tanto la verdad de todas las proposiciones jurídicas puras, es decir aquellas cuya verdad sólo depende de la existencia de determinadas normas jurídicas cuanto la verdad de todas las proposiciones jurídicas aplicadas, cuya verdad depende de la existencia de determinadas normas jurídicas y de la verdad de determinadas proposiciones fácticas. Podemos explicar la distinción con el ejemplo de KELSEN: la verdad de «Según el derecho, si alguien mata a otro debe ser castigado a reclusión perpetua» depende sólo de la existencia de una norma jurídica, es una proposición jurídica pura, la verdad de «Según el derecho, Schuster debe ser castigado a reclusión perpetua» depende de la existencia de la norma jurídica y de la verdad de que Schuster haya matado a otra persona, es una proposición jurídica aplicada ${ }^{10}$. Pero RAZ rechaza ambas versiones de la tesis con el argumento de que ni creemos ni estamos dispuestos a aseverar todo aquello que es consecuencia lógica de nuestras creencias o de nuestras aserciones; como enfáticamente sostiene (1994: 228): «People do not believe in all that is entailed by their beliefs». Aquí no indagaré sobre la posición raziana, que como es sabido es vicaria de su concepción de la autoridad, y me conformaré con rescatar esta distinción que es valiosa para lo que más adelante plantearé.

Sin embargo, siguiendo la estela de RAZ, MARMOR (2001: 69-70) ha dado un argumento ulterior para rechazar tanto la versión débil como la fuerte de la incorporación. Conforme a MARMOR, para suponer que las normas implicadas por las normas válidamente promulgadas son también válidas, hay que suponer que el sistema promulgado de normas es coherente y como esta le parece una implausible posición rechaza de plano la tesis de la incorporación. No queda muy claro por qué le parece a MARMOR que sólo un sistema consistente de normas puede implicar otras normas. Tal vez porque piensa que un sistema inconsistente permite, en virtud de la regla ex falso quodlibet, que cualquier norma sería parte de dicho sistema ${ }^{11}$. Sin embargo, como bien arguyen NAVARRO y RODRíGUEZ (2014, cap. 6) un conjunto inconsistente de normas es un conjunto de normas claramente defectuoso, sin embargo es un conjunto que tiene consecuencias lógicas, más aún para definir la noción de contradicción entre normas es necesario presuponer la noción de implicación entre normas.

Hay, con todo, un punto de verdad en la perplejidad que produce que cualquier norma pueda derivarse de un conjunto inconsistente. La perplejidad tal vez pueda ser explicada por la siguiente razón: cualquier conjunto de premisas (sean normas o no) implica muchas normas irrelevantes. Así, por ejemplo, de la norma «Los que matan a otro deben ser castigados con la sanción $\mathrm{S} \gg$ se puede obtener como consecuencia «Los que matan a otro vestidos de azul deben ser castigados con la sanción S» o bien «Los que matan a otro deben ser castigados con la sanción $\mathrm{S}$ o premiados con unas vacaciones en la isla de Menorca». Es un problema bien conocido de la comunicación y por ello GRICE (1975: 46) incluyó la máxima de relevancia («Be relevant») entre sus máximas de la conversación. Un desarrollo de dicha noción puede evitar estas consecuencias contra-intuitivas y mostrar que, por ejemplo, un sistema inconsistente es aquel que carece de consecuencias relevantes ${ }^{12}$.

\footnotetext{
10 RAZ, 1994: 230-231. La distinción ya se hallaba en RAZ, 1980: 49, 218.

11 Un argumento semejante se halla en ATIENZA, 1991: 34-35.

12 Desarrollé esta idea con este objetivo en MoRESO, 1996.
} 
Tal vez esta última sugerencia esté en armonía con una idea de RAZ (1994: 214) en donde se refiere a la posibilidad de que una concepción más compleja pueda ser contemplada como una combinación de la tesis de las fuentes y la tesis de la incorporación. Sea como fuere, para establecer las condiciones de verdad de las proposiciones jurídicas, podemos aceptar la versión débil de la tesis de las fuentes, restringida a las consecuencias lógicas relevantes. Sin embargo, y de esto es de lo que voy a ocuparme a continuación, la versión fuerte necesita restricciones más poderosas: como ya veíamos en la introducción, hay proposiciones verdaderas que no parecen candidatos a combinarse con las proposiciones jurídicas que se refieren a normas válidas para obtener otras proposiciones jurídicas y hay proposiciones falsas que parecen candidatos idóneos.

\section{EL OPERADOR «SEGÚN EL DERECHO»}

En muchas oraciones es posible reemplazar un nombre propio por una descripción definida, por ejemplo, que se refieran al mismo individuo y preservar el valor de verdad de la oración. Lo mismo sucede con los predicados cuando son sustituidos por predicados que tienen su misma extensión. Son sustituibles salva veritate. Por ejemplo, el valor de verdad de la siguiente oración

[1d] Cervantes escribió el Quijote

no cambia si sustituimos «Cervantes» por la descripción definida «el manco de Lepanto»

[2d] El manco de Lepanto escribió el Quijote.

Ahora bien, si a una oración como [1d] prefijamos un operador intensional, que expresa una actitud proposicional epistémica, modal, de ficción, etc. Entonces ya no preservamos la verdad mediante la substitución. Pensemos en el siguiente ejemplo:

[3d] Sé que Cervantes escribió el Quijote

puede ser verdadero y, no obstante, yo puedo desconocer que CERVANTES perdió una de sus manos en la famosa batalla de Lepanto contra los turcos y entonces ser falso que

[4d] Sé que el manco de Lepanto escribió el Quijote.

Es por ello que nuestra noción de creencia o de saber no respeta la versión más fuerte del principio de clausura epistémica, según el cual el conjunto de lo que sabemos (o creemos) es un conjunto cerrado bajo la noción de consecuencia lógica con el conjunto de todas las verdades. Esta versión es ampliamente rechazada (vid., por todos, LUPER, 2012). Más aceptada es la versión según la cual: si x sabe que p y x sabe (o, para algunos, incluso x cree) que $\mathrm{p}$ implica $\mathrm{q}$, entonces $\mathrm{x}$ sabe que $\mathrm{q}^{13}$. Aunque sospecho que, en resumidas cuentas, todos estos problemas asociados a la intensionalidad proceden de una caracterización adecuada de la modalidad, desarrollaré las ideas para el

13 Una famosa objeción a esta versión del principio en NOZICK, 1981: 227-229, según NoziCK yo sé que estoy sentado esta tarde de mayo frente al ordenador, sé que si estoy sentado esta tarde de mayo frente al ordenador entonces no soy un cerebro en una cubeta, sin embargo no sé que no soy un cerebro en una cubeta, ya que una de las condiciones del saber es que yo sé que p si p fuera falso, entonces yo no lo creería y que no soy un cerebro en una cubeta lo creería aunque lo fuese. 
operador jurídico a partir de un contexto que le es más cercano. Me refiero al contexto de la ficción ${ }^{14}$. Un problema semejante al de los gansos en Cuaresma para el derecho de la Iglesia católica medieval, fue claramente presentado en un seminal artículo de David LEWIS (1978). LEWIS tomó como referencia el personaje del famoso detective Sherlock Holmes en las novelas de Conan DoYlE y entonces mostró este tipo de dificultades con el siguiente ejemplo:

[1e] En la ficción Conan Doyle, Sherlock Holmes vive en Baker Street 221B.

[2e] En Baker Street 221B hay una oficina bancaria ${ }^{15}$.

[3e] En la ficción Connan Doyle, Sherlock Holmes vive en una oficina bancaria.

No todo lo que es verdad en nuestro mundo real puede ser combinado con lo que es verdad en la ficción para obtener nuevas verdades en la ficción. Hay que hallar algún modo de restringir las combinaciones posibles para establecer las condiciones de verdad de las proposiciones referidas a la ficción ${ }^{16}$. Sin embargo, no tenemos dificultades para aceptar el siguiente razonamiento ${ }^{17}$ :

[1f] En la ficción Conan Doyle, Sherlock Holmes vive en Londres.

[2f] Londres es una ciudad del Reino Unido.

[3f] En la ficción Connan Doyle, Sherlock Holmes vive en el Reino Unido.

Entonces el problema es establecer adecuadamente las condiciones de verdad de las proposiciones expresadas por oraciones como:

[F] En la ficción $\mathrm{f}, \varphi$,

si la ficción son las novelas de DOYLE, y $\varphi$ está en el lugar de «Sherlock Holmes vive en Gran Bretaña», entonces obtenemos una proposición verdadera. Sin embargo, si $\varphi$ está en el lugar de «Sherlock Holmes vive en un banco» obtenemos una proposición falsa. La causa de esta divergencia reside en el hecho de que las descripciones de los mundos de ficción contienen implícitamente muchas proposiciones que, por una parte, son compatibles con lo descrito en la ficción y, por otra, son representaciones verdaderas de nuestro mundo. En las novelas de DOYLE, el detective y el doctor Watson son seres humanos, la sangre corre por sus venas, tienen corazón y riñones, etc. Ahora bien, en las novelas de DOYLE, Baker Street no es como la calle del Londres de su época, al menos porque allí habita una persona que no habita en el Londres real.

Los autores (LEWIS, 1978; CURRIE, 1990; WALTON, 1990; BONOMI-ZuCCHI, 2003; GARCÍA-CARPINTERO, 2007, por ejemplo) que se han dedicado a esta cuestión han tratado de dar las condiciones de verdad de proposiciones que instancian [F]. Tratan de mostrar que las proposiciones verdaderas de acuerdo con la ficción son aquellas

\footnotetext{
14 Más sobre la comparación entre las proposiciones referidas a la ficción y las proposiciones referidas al derecho en MORESO-CHILOVI (ms.).

15 De hecho, ya lo advierte LEWIS, en la época en que las novelas fueron escritas y publicadas Baker Street no tenía números tan altos, es decir no había una casa en Baker Street con ese número.

16 Este es el intento del trabajo de LEWIS comentado, que ha sido desarrollado por el sugerente trabajo de BONOMI-ZuCHI (2003).

17 El ejemplo es de MARMOR (2014: 81). MARMOR se plantea el problema referido a las proposiciones jurídicas, sin embargo sugiere que en el razonamiento jurídico todas las proposiciones son prefijadas por el operador «Según el derecho» y, entonces, no tiene el problema de la combinación de dichas proposiciones con las proposiciones no prefijadas que describen nuestro mundo real. No seguiré esta vía principalmente porque si el derecho pretende regular los comportamientos del mundo real, entonces debe tomar en cuenta las descripciones verdaderas de este mundo.
} 
relativas a un mundo en el cual ocurre todo lo que se describe en la novela y, en lo demás, pertenece al conjunto de mundos más cercanos al mundo real compatible con la primera restricción. Es obvio que las ficciones no describen un solo mundo posible, dado que la noción de mundo posible presupone que cualquier proposición es o bien verdadera o bien falsa respecto de tal mundo ${ }^{18}$. Las novelas de Conan DoYLE son compatibles con que el doctor Watson fuese de uno u otro grupo sanguíneo, todos los mundos en que Watson es del grupo A o del B o... son compatibles con la ficción ${ }^{19}$.

Sin embargo, hay al menos dos razones más para restringir las condiciones de verdad de las proposiciones jurídicas. Una guarda relación con las convenciones propias de la ficción (BONOMI-ZuCCHI, 2003), en su artículo de 1978 LEWIS ya llamaba la atención acerca de que si leemos una historia de dragones y princesas, aunque la historia no dice nada acerca de que el dragón echa fuego por la boca, las convenciones de la ficción nos llevan a concluir que sí lo hace. El otro aspecto tiene que ver con las creencias falsas del autor de la ficción y de la comunidad de la cual forma parte, creencias que conforman lo que STALNAKER (2002) llamó el common ground. En algunas ocasiones, una proposición falsa puede formar parte del trasfondo común (common ground) que compartimos en una conversación. Supongamos que después de un seminario con una colega invitada, SAMANTHA, surge la cuestión entre CHIARA y yo acerca de si José Luis, otro colega, que nosotros creemos que está impartiendo una clase, va a acudir puntualmente a la cena que tenemos en 30 minutos. CHIARA sostiene que sí y yo sostengo que llegará media hora tarde. Supongamos también que es falso que José Luis esté dando clase, en realidad está en un congreso en Princeton (y, claro, no puede venir a la cena en Barcelona). Sin embargo, no podemos atribuir sentido a nuestra discrepancia, ni si quiera establecer en qué condiciones serían verdaderas nuestras proposiciones sin suponer que la proposición falsa, según la cual José Luis está en clase, es parte del trasfondo común. Pues bien, esto ocurre también en la ficción: por ejemplo el pasaje de la Odisea en el que los marineros de Ulises comen el fruto del loto en la isla de los lotófagos (tal vez la isla de Yerba) ni siquiera puede comprenderse sin suponer la creencia común y falsa (de Homero como autor del poema y de su comunidad relevante), conforme a la cual comer el fruto del loto produce la pérdida de la memoria.

Esto es, las proposiciones referidas a una ficción son verdaderas si reproducen lo dicho en la ficción y lo implicado por ella, que incluye no sólo lo lógicamente implicado por lo dicho en la ficción, sino también las convenciones del género, las creencias compartidas que la ficción presupone y las descripciones verdaderas de nuestro mundo real compatibles con lo anterior.

Pues bien, algo semejante ocurre en el derecho. Llamemos proposiciones jurídicas a todas aquellas expresadas mediante enunciados prefijados por el operador «Según el derecho». Llamemos proposiciones no-jurídicas a todas aquellas expresadas median-

18 Prescindamos ahora de la posibilidad de proposiciones que carezcan de valor de verdad para tratar los casos de indeterminación.

19 Por ello podríamos decir que en este caso «Según la ficción f, el doctor Watson es del grupo sanguíneo B» está indeterminada y, por ende, carece de valor de verdad. Que carece de valor de verdad aquí equivale a que esta proposición es verdadera en alguna de las compleciones de la ficción y falsa en otras, como quiere el superevaluacionismo (vid., para una presentación, KEEFE, 2008). 
te enunciados asertivos no-prefijados. Y expresemos su forma canónica del siguiente modo:

\section{[D] Según el derecho D, $\varphi$.}

$\varphi$ puede estar en el lugar o bien de un enunciado deóntico (como «los ciudadanos mayores de edad tienen derecho a votar en las elecciones generales») o puede ser la expresión de una regla constitutiva (como «los mayores de 18 años son mayores de edad») ${ }^{20}$. Las reglas constitutivas conectan unas reglas con otras en la red del sistema jurídico, son como sus nodos, de los dos ejemplos anteriores podemos concluir que «Según el derecho D, los mayores de 18 años tienen derecho a votar en las elecciones generales». Podemos denominar a estas conexiones entre reglas relaciones internas ${ }^{21}$. Porque dado que el derecho pretende regular el comportamiento humano para alcanzar determinados fines que consideramos valiosos, entonces las proposiciones jurídicas más relevantes son las que nos informan de aquello que debemos hacer, de aquello que tenemos prohibido y de aquello que estamos autorizados a llevar a cabo. Las reglas constitutivas son conexiones internas del sistema para alcanzar dichos resultados. Por ejemplo, un español mayor de edad puede otorgar testamento escribiendo de puño y letra, firmando y fechando sus últimas voluntades, lo que se conoce como testamento ológrafo porque así lo establece la regla constitutiva contenida en el art. 688 del Código Civil.

Es más, el derecho pretende influir en nuestro mundo real, pretende que nuestro mundo real cambie y se convierta en el (conjunto de) mundos deónticamente ideales que sus normas prescriben ${ }^{22}$. Según parece, estos mundos deónticamente ideales han de ser mundos accesibles desde nuestro mundo real. Pero esta plausible afirmación debe ser algo restringida, como ocurre en la ficción. El conjunto de mundos deónticamente ideales debe ser construido a partir de un mundo ligeramente diferente del mundo real, para dar cuenta de dos aspectos claves: de las convenciones del derecho (establecidas por sus reglas constitutivas) y de las creencias falsas que forman parte del common ground de los miembros de la comunidad relevante en ese sistema jurídico.

Las convenciones en el derecho vienen dadas por sus reglas constitutivas. Aquí el supuesto más claro es, como hemos visto al principio, el de las ficciones jurídicas. En estos casos creo que debemos aceptar que si «según el derecho D, $\varphi$ », entonces obtenemos « $\varphi »$. Pero, ¿qué ocurre si sabemos que $\varphi$ es falsa, como ocurre siempre en el caso de las ficciones, y a veces, en el caso de las presunciones? Pensemos que sabemos que el padre de Raimundo no es el marido de la madre de Raimundo, pero rige el Code Napoléon. En este caso, si aceptamos que las proposiciones verdaderas formen parte de nuestros razonamientos jurídicos produciríamos una contradicción.

20 No desarrollaré esta cuestión que, sin embargo, me parece bien asentada en la teoría jurídica actual (vid., por todos, ATIENZA y RUIZ MANERO, 1996) y en la teoría de las instituciones sociales en general (SEARLE, 1995). Tampoco me detendré en otra complicación, los enunciados que instancian [F] deberían tener también un parámetro temporal: «Según el derecho D en t» (puede verse, por ejemplo, HeRNÁNDEZ MARÍN y SARTOR, 1999).

21 La denominación se debe a RAZ (1980: 145-146, 169-172) y está en el trasfondo del famoso trabajo de Alf Ross (Ross, 1957).

22 Para una buena presentación de la idea de mundo deónticamente ideal y, también, de sus problemas en los que no puedo detenerme ahora, HANSSON, 2006. 
Para evitarlo creo que es bueno distinguir entre creer y aceptar $^{23}$. Creer que p consiste en una disposición a tomar como verdadera la proposición p, aceptar que p consiste en una disposición a adoptar como premisa la proposición p en determinado contexto argumentativo. Debe apreciarse, entonces, que podemos creer una proposición y no aceptarla y, también, podemos aceptar una proposición aunque no la creamos, incluso aunque sepamos que es falsa. Esta es la primera restricción a la aceptación de proposiciones verdaderas en nuestros razonamientos jurídicos, es decir, si «según el derecho D, $\varphi »$; entonces $\varphi$ ha de ser aceptada aunque sea falsa. Las proposiciones no-jurídicas de nuestros razonamientos jurídicos deben ser, verdaderas o falsas, aceptables.

A continuación, vamos a analizar el segundo y relevante supuesto de proposiciones falsas que han de ser aceptadas, de las creencias falsas que forman parte de los presupuestos comunes de los miembros relevantes de determinada comunidad humana.

\section{LAS CONDICIONES DE VERDAD DE LAS PROPOSICIONES JURÍDICAS: BALLENAS, TOMATES Y HONGOS}

Ocurre en el derecho algunas veces que determinadas consecuencias normativas, que son queridas por las autoridades, presuponen creencias falsas. Veámoslo con algunos ejemplos.

En 1818, en Maurice v. Judd, un inspector del uso de los aceites, Maurice, demandó a Judd reclamándole una cantidad de dinero por haber comprado tres barriles no inspeccionados de aceite de ballena. Judd no negó los hechos sino que arguyó que la legislación del estado de Nueva York prescribía la inspección de los aceites de pescado y por tanto no se aplicaba a los barriles que él compró, que eran barriles de aceite de ballena. El jurado del tribunal de Nueva York rechazó su pretensión, y estableció que a los efectos de la ley de inspección, el aceite de pescado incluía el aceite de ballena ${ }^{24}$. En esos años precisamente se desarrollaba la zoología y las taxonomías iban variando, Lineo había clasificado en 1756 a las ballenas como peces, y en la edición de 1758 cambió y las calificó, ya acertadamente, como mamíferos. Pero, olvidemos ahora esta oscilación y olvidemos por un momento también las relevantes cuestiones de filosofía del lenguaje referidas al significado de las expresiones de género natural y sus consecuencias para esta polémica (aunque si «mamífero» se toma como un designador rígido y «pez» también sin incluir a los mamíferos, entonces una proposición según la cual las ballenas son peces es falsa en todos los mundos posibles) ${ }^{25}$. Supongamos que todos los miembros de la comunidad relevante creían falsamente cuando la legislación sobre inspección de aceites fue dictada que las ballenas son peces, entonces en ese momento esta proposición no-jurídica es aceptable. Supongamos que más adelante los miembros de la comunidad relevante se dan cuenta de su error, se retractan y aceptan que las ballenas son mamíferos y no peces ${ }^{26}$. Creo que, en este caso, la pregunta

23 Siguiendo a COHEN, 1989.

24 La historia aparece magníficamente contada en BURNETT, 2007.

25 Que han dado lugar a un reciente e instructivo debate de filosofía del lenguaje, SANSBURY, 2014; PHILIPS, 2014.

26 Hay reciente literatura sobre los desacuerdos y los cambios de opinión posteriores, por ejemplo, MACFARLANE, 2014: 11-15, 108-111, FERRARI y ZEMAN, 2014. 
debe ser si los miembros de la comunidad relevante estarían dispuestos o no a retirar la obligación de la inspección del aceite de ballena, cambiando la legislación si fuese preciso. Si la respuesta es que no estarían dispuestos a retirar esta consecuencia normativa, entonces, la proposición jurídica «Según el derecho, el aceite de ballena debe ser inspeccionado» es verdadera y precisamos, mientras no se cambie la legislación, de la proposición falsa - pero aceptable_- según la cual el aceite de ballena es aceite de pescado. El razonamiento es el siguiente:

[1g] Según el derecho de Nueva York, el aceite de pescado debe ser inspeccionado.

[2g] Las ballenas son peces.

[3g] Según el derecho de Nueva York, el aceite de ballena debe ser inspeccionado.

Hay otros casos jurídicos que pueden avalar esta conclusión parcial ${ }^{27}$. En 1883, en los Estados Unidos determinada legislación fiscal (Tariff Act of March 3, 1883) establecía una carga impositiva a la compraventa de verduras, pero no a la de frutos. De modo que se produjo una controversia acerca de si los tomates han de ser clasificados como verduras o como frutos. Es obvio que, desde el punto de vista botánico, los tomates son un tipo de fruto y, también es conocido, que desde el punto de vista culinario acostumbramos a servir los tomates junto con otras verduras, en la ensalada por ejemplo y no como postre, y a menudo los tomates se hallan en las tiendas de comestibles junto con las verduras y no con los frutos. De acuerdo con la teoría causal de la referencia aplicada a los términos usados en los textos legales, deberíamos concluir que los tomates son frutos y que por tanto están exentos del impuesto. Sin embargo, la Corte Suprema de los Estados Unidos en 1893 sostuvo que de acuerdo con el uso habitual de los términos y a los únicos efectos de la interpretación de la ley en disputa, los tomates son considerados verduras ${ }^{28}$. Estas fueron las palabras del juez Gray, con una decisión tomada por unanimidad:

Botánicamente hablando, los tomates son el fruto de una planta, como los son los pepinos, los calabacines, las judías y los guisantes. Pero en el lenguaje común de la gente, sea de los vendedores o de los consumidores de estos productos, todos son verduras que se cultivan en los huertos domésticos y que cocinados o crudos, son como las patatas, las zanahorias, la chirivía, los nabos, la remolacha, la coliflor, la col, el apio y la lechuga, que usualmente se sirven para cenar con o después de la sopa, el pescado o la carne que constituye la parte principal de la comida y no, como los frutos generalmente, el postre.

Lo que parece abonar mi conclusión, el Tribunal Supremo aún reconociendo que los tomates son frutos, muestra sus razones relativas a la calificación del comportamiento, según las cuales los tomates a los efectos de la legislación fiscal del caso, han de ser considerados como verduras.

Veamos otro supuesto recalcitrante también de la botánica: la legislación penal, la ley de tráfico de estupefacientes - que contiene un anexo con las sustancias prohibidas - alemana castigaba el tráfico de plantas alucinógenas, algunos de los defensores de traficantes con hongos alucinógenos arguyeron que los hongos no son, botánicamente, plantas, por lo que no podían ser penados por su tráfico y conforme a ello el Oberlandgericht (Tribunal Superior) de Coblenza absolvió a unos acusados de este

27 De los que me ocupé en J. J. MoreSO, 2010.

28 Vid. Nix v. Hedden (149 US 304, 1893). 
delito. En octubre de 2006, sin embargo, el Bundesgerichtshof (Tribunal Supremo Federal de Alemania) revoca en casación esta decisión con argumentos muy similares a los que cien años antes había usado el Tribunal Supremo estadounidense ${ }^{29}$. Estos son los argumentos fundamentales del Tribunal:

En verdad se reconoce en la biología que los hongos se ubican como un grupo independiente de organismos junto a las plantas (verdes) [...] en definitiva, se compran generalmente hongos también en fruterías y verdulerías. La filiación [entre plantas y hongos] es comprobada mediante una búsqueda en Internet, que está a disposición de cualquiera para la publicación de textos propios y, por tanto, puede brindar una amplia información sobre el espectro general del actual uso del lenguaje. Precisamente allí pueden encontrarse algunas páginas webs en las que se indica que los hongos — desde un punto de vista científico- no son como las plantas, aunque también allí se añade que los hongos siempre son clasificados de manera errónea (esto es, en el lenguaje ordinario) a las plantas.

Al parecer, aunque la botánica es la mejor teoría de la que disponemos para el reino vegetal y que sus clasificaciones naturales hacían de los tomates frutos y, por ello, no incluidos en la referencia de la expresión «verduras» y los hongos son un grupo de organismos diferenciado de las plantas; no obstante la comunidad jurídica relevante no está dispuesta a revisar sus calificaciones normativas por ello.

Sin embargo, no siempre es así. Algunas veces el abandono de una creencia y su sustitución por otra, verdadera, comporta la aceptación por la comunidad relevante del cambio de consecuencias normativas. Hace ya un tiempo se consideraba que la muerte de una persona sucedía cuando dejaba de respirar y de funcionar su corazón. Más adelante la ciencia médica ha establecido que la muerte se produce cuando cesa la actividad cerebral ${ }^{30}$. Pensemos en un razonamiento jurídico como el siguiente:

[1h] Según el derecho D, un cuerpo humano sólo puede ser preparado para un trasplante de órganos después de su muerte.

[2h] La muerte sucede cuando cesa la actividad cerebral.

[3h] Según el derecho D, un cuerpo humano sólo puede ser preparado para un trasplante de órganos después de que cese su actividad cerebral.

Para que este razonamiento jurídico sea correcto, debemos suponer algo como que los miembros de la comunidad jurídica relevante están dispuestos a aceptar los cambios normativos consecuencia de su cambio de creencias. En este caso parece claro que todos aceptarían que el derecho no permite el trasplante de órganos cuando el corazón ha dejado de latir, pero hay todavía actividad cerebral. Son casos en los que en nuestro contexto de interpretación ${ }^{31}$ los cambios o bien en el mundo o bien en nuestro conoci-

29 Vid. BGH, NJW, 2007, 524-526. Vid. también MONTIEL y RamírEZ, 2010.

30 Un ejemplo preferido de MOORE, 1985. Agradezco a Lorena RAMírEZ que me recordara este ejemplo tan pertinente para lo que deseo mostrar.

31 CAPPELEN, 2008, defiende, con referencia particular al derecho, que el contexto en el que una disposición jurídica es interpretada puede ser distinto en distintos momentos, comenzando por el de su promulgación. CAPPELEN añade, muy razonablemente, que cuando el legislador introduce términos genéricos como «peligroso» o «sospechoso» deja un amplio margen a los intérpretes para que establezcan lo que cae en la referencia de dichos términos en los contextos apropiados: una mochila abandonada en un aeropuerto puede ser tildada de peligrosa, pero tal vez no en un sendero para excursionistas. Por ejemplo, el art. 606.2 de la Ley de Enjuiciamiento Civil del año 2000 establece que son inembargables «los libros e instrumentos necesarios para el ejercicio de la profesión, arte u oficio a que se dedique el ejecutado, cuando su valor no guarde proporción con la cuantía de la deuda reclamada»; aunque un Ipad (dado que todavía no existían) no era inembargable en 
miento de él (como en este caso) conducen a cambios en las consecuencias normativas de nuestro sistema jurídico, cambios que estamos dispuestos a aceptar ${ }^{32}$.

Estos son, según mi opinión, los casos claros. En otros muchos supuestos la controversia será mayor. Supongamos el siguiente razonamiento:

[1i] Según el derecho D, están prohibidas las penas crueles e inusuales.

[2i] La pena de muerte es cruel.

[3i] Según el derecho D, la pena de muerte está prohibida.

Este es un razonamiento muy controvertido. Por una parte, la verdad de la premisa [2i] requiere alguna metaética no totalmente escéptica acerca de un espacio de objetividad para la moral. Por otra parte, alguien puede argüir con plausibilidad que, por ejemplo, cuando la enmienda octava de la Constitución de los Estados Unidos fue promulgada con este contenido los miembros de la comunidad jurídica relevante creían en la verdad de la proposición «la pena de muerte no es cruel». Pero si ahora, aceptando este espacio de objetividad para la moral, nos convencemos de que esa era una proposición falsa, tal vez podemos conjeturar que los miembros de la comunidad relevante habrían mantenido la prohibición en dicho caso, ampliándola por tanto a la pena de muerte o bien que lo relevante es nuestro propio juicio, en un contexto de interpretación diferente, acerca de la crueldad de la pena de muerte ${ }^{33}$.

Con estas ideas tal vez podamos dar las dos siguientes definiciones:

[DEF I] Una proposición no-jurídica $\varphi$ es aceptable si:

(i) $\varphi$ es falsa, pero es verdad que según el derecho, $\varphi$.

(ii) $\varphi$ es falsa, pero $\varphi$ forma parte de las creencias que son presupuesto común de la comunidad relevante y los miembros de dicha comunidad no estarían dispuestos a retirar las consecuencias normativas que se siguen de $\operatorname{aceptar} \varphi$, aunque supieran que $\varphi$ es falsa.

(iii) $\varphi$ es verdadera, en el resto de los casos.

[DEF II] Una proposición jurídica [D]: «Según el derecho, $\varphi$ »s verdadera si:

(i) $\varphi$ es un contenido normativo explícito (promulgado por un autoridad jurídica)

(ii) $\varphi$ es un contenido normativo implícito relevante (implicado por lo promulgado por una autoridad jurídica).

(iii) $\varphi$ es una consecuencia lógica relevante del contenido normativo explícito e implícito y del conjunto de proposiciones no-jurídicas aceptables ${ }^{34}$.

el momento de la promulgación de la ley, pero tal vez podría argüirse en 2015 a favor de su inembargabilidad cuando el deudor sea un abogado. Vid. MORESO, 2014.

32 En un interesante trabajo PERRY (2011) distingue precisamente para estos supuestos el modelo del cambio del modelo del conocimiento.

33 Es el argumento de PERRY, 2011. Una discusión sobre PERRY que tiene en cuenta las dos posibilidades anteriomente mencionadas, es decir que los miembros de la comunidad relevante estén dispuestos a cambiar las consecuencias normativas en virtud de la nueva verdad adquirida, como en las penas crueles o de la muerte, o no lo estén, como en el caso de las ballenas y de los tomates, en AsgeIRSson, 2012. Scott J. SHAPIRO (SHAPIRO, 2009), en cambio, parece pensar que aunque sea verdad que los castigos crueles están prohibidos, bajo está descripción, y sea verdad que la pena de muerte es cruel, no puede decirse que jurídicamente la pena de muerte está prohibida. No alcanzo a comprender las razones de esta restricción tan absoluta a la descripción de las acciones que estableció el legislador, sería como sostener extrañamente que aunque una norma prohíbe cazar animales salvajes y los leones son animales salvajes, no puede decirse que jurídicamente está prohibido cazar leones.

34 En las cláusulas (ii) y (iii) de esta segunda definición, «relevante» puede ser entendido con la ayuda de MORESO (1996): para la lógica proposicional, una consecuencia lógica es relevante en relación con sus pre- 
Con ello mostramos que la tesis de la incorporación debe ser rectamente entendida. La tesis no establece la clausura bajo la noción de consecuencia lógica de las normas de un sistema jurídico y las proposiciones no-jurídicas verdaderas. Dicha clausura tiene el límite de las consecuencias relevantes y, hay que advertirlo, la noción de consecuencia relevante no es monotónica. Y, más importante, tiene el límite de sustituir, para las proposiciones no-jurídicas, la verdad por la aceptabilidad. Este es un modo de comprender las restricciones que surgen de la semántica y la pragmática del operador intensional «Según el derecho».

\section{CODA: LAS CONVENCIONES Y LA MORALIDAD}

Dos comentarios finales que tal vez ayuden a completar lo defendido en este trabajo.

En primer lugar no habría de resultar sorprendente que las convenciones, en la ficción y en el derecho, determinen y constriñan nuestros razonamientos jurídicos. Así sucede también en nuestras conversaciones ordinarias. Supongamos que una tarde mi hija Julia de quince años descubre entre sus cosas un viejo peluche que le gustaba cuando era pequeña, unos amigos le preguntan de dónde lo sacó y ella contesta: «Me lo trajeron los Reyes Magos cuando tenía cinco años». Todos comprendemos lo que dice y son las convenciones de nuestro lenguaje de adultos los que hacen aceptable esta oración en la conversación, aunque sea falsa literalmente. Todos sabemos que es falsa, pero puede funcionar como premisa de argumentos en este contexto. Alguien podría objetar que esta oración debe ser parafraseada y sustituida por otra como «Me lo regaló alguien cercano para la fiesta de los Reyes Magos de cuando tenía cinco años», como alguien podría decir que «Menorca está en Londres» debe ser entendida como la paráfrasis «La jurisdicción sobre los residentes en Londres de la Court of Common Pleas se extiende a los residentes en Menorca». Pero esto comportaría abandonar las ficciones, puesto que en este enunciado no hay ficción alguna. Y, por razones pragmáticas, al igual que en el caso de los Reyes Magos, es conveniente preservar esta dimensión de nuestro lenguaje. Una dimensión que, una vez comprendida, es inocua y es, además, capaz de mostrar que un día, cuando éramos niños, teníamos creencias falsas. Como bien arguye STALNAKER (2002: 702) podría ser que en algunos casos la mejor explicación del valor de verdad de algunas oraciones consista en sostener que se ha presupuesto algo que es falso.

La segunda observación sigue una sugerencia, referida a la ficción, de WALTON (1994). WaLTON comienza con un pasaje de Hume (1777) en su estudio sobre el estándar del gusto: mientras le parecen totalmente aceptables en las obras de ficción del pasado las referencias a claras falsedades (como princesas acarreando el agua de la fuente dice HumE) o, incluso, los errores que restan, nos dice, poco valor a estas

misas si ninguna variable proposicional puede ser sustituida por cualquier otra salva validitate (es una idea de SCHURZ, 1991); para la lógica deóntica (necesaria si $\varphi$ está en el lugar de un enunciado deóntico) la anterior idea ha de completarse con la siguiente: las consecuencias deónticas que no son soluciones maximales (que no determinan tanto la acción como la omisión) no son derivables de otras consecuencias que son máximales; por ejemplo si según un código de normas está prohibido fumar, entonces no debemos deducir relevantemente que según dicho código está permitido no fumar. 
composiciones, las diferencias morales son otra cosa: no debemos ni tolerar ni adoptar las «ideas de moralidad y decencia» que son repugnantes para nosotros. WALTON está de acuerdo con Hume y añade (1994: 35): «Mi sugerencia fue, en breve, que cuando interpretamos los trabajos literarios u otras obras artísticas estamos menos dispuestos a permitir que los mundos ficcionales de las obras de arte se separen del mundo real en cuestiones morales que en cuestiones no-morales». No vemos problema en imaginar una sociedad en donde los seres humanos pierden la memoria por comer la flor del loto, pero tenemos más dudas en leer una novela situada en una sociedad esclavista como si la esclavitud fuese aceptable (aunque lo fuese para el autor de la novela y para sus personajes), como las tenemos para adoptar el punto de vista de la venganza despiadada en La Ilíada para seguir con HOMERO.

La idea es controvertida y no será desarrollada aquí, pero mi conjetura es que algo semejante ocurre con el derecho. Podemos adoptar la ficción de que las llaves auténticas perdidas por su dueño son falsas, podemos aceptar que cuando el legislador se refería a las verduras incluía los tomates, pero no podemos aceptar que el genocidio o el apartheid sean correctos aunque el derecho de una comunidad lo establezca o presuponga. El desarrollo de las consecuencias de esta sugerencia habrá de quedar para otra ocasión.

\section{BIBLIOGRAFÍA}

Alchourrón, C. E., y Bulygin, E., 1971: Normative Systems, New York-Wien: Springer.

Asgeirsson, H., 2012: «Textualism, Pragmatic Enrichment, and Objective Communicative Content» (September 6, 2012), Monash University Faculty of Law Legal Studies Research Paper núm. 2012/21. Available at SSRN: http://ssrn.com/abstract=2142266.

AtienZA, M., 1991: Las razones del Derecho, Madrid: Centro de Estudios Constitucionales.

AtienZA, M., y Ruiz Manero, J., 1996: Las piezas del Derecho, Barcelona: Ariel.

BerTO, F., 2013: «Impossible Worlds», en E. N. ZalTa (ed.), The Stanford Encyclopedia of Philosophy (Winter 2013 Edition), URL: http://plato.stanford.edu/archives/win2013/entries/ impossible-worlds/.

Bonomi, A., y Zucchi, S., 2003: «A Pragmatic Framework for Truth in Fiction», Dialectica, 57: 103-120.

Bulygin, E., 2006: El positivismo jurídico, México: Fontamara.

Burnett, D. G., 2007: Trying Leviathan: The Nineteenth-Century Court Case That Put the Whale on Trial and Challenged the Order of Nature, Princeton: Princeton University Press.

CAPPELEN, H., 2008: «The Creative Interpreter: Content Relativism and Assertion», Philosophical Perspectives, 22: 23-46.

Caracciolo, R., 1988: Sistema jurídico. Problemas actuales, Madrid: Centro de Estudios Constitucionales.

CoHen, L. J., 1989: «Belief and Acceptance», Mind, 98: 367-389.

CurRIE, G., 1990: The Nature of Fiction, Cambridge: Cambridge University Press.

Dworkin, R., 2006: Justice in Robes, Cambridge, Mass.: Harvard University Press.

FERrari, F., y ZEMAN, D., 2014: «Radical Relativism, Retraction and "Being at Fault"», en

F. Bacchini, S. Caputo y M. Dell’Utri (eds.), New Frontiers in Truth, New Castle upon Tyne: Cambridge Scholars Publishing, cap. 6. 
Friedman, J. B., y FigG, K. M. (eds.), 2000: Trade, Travel, and Exploration in the Middle Ages. An Encyclopedia, London: Routledge.

GARCÍA CARPINTERO, M., 2007: «Fiction-Making as a Gricean Illocucionary Type», The Journal of Aesthetics and Art Criticism, 65: 203-216.

Grice, P., 1975: «Logic and Conversation», en P. COLE y J. Morgan (eds.), Syntax and Semantics 3: Speech Acts, New York: Academic Press, 41-58.

Hansson, S. O., 2006: «Ideal Worlds: Wishful Thinking in Deontic Logic», Studia Logica: An International Journal for Symbolic Logic, 82: 329-336.

HERnÁndez MARín, R., 1984: El derecho como dogma, Madrid: Tecnos.

— 1986: «Ficciones jurídicas», Doxa, 3: 141-147.

Hernández Marín, R., y SARTOR, G., 1999: «Time and Norms: A Formalisation in the Eventcalculus», en Proceedings of the Seventh International Conference on Artificial Intelligence and Law (ICAIL), New York: ACM, 90-100.

Himma, K. E., 2002: «Inclusive Legal Positivism» en J. L. Coleman y S. J. SHaPIRO (eds.), The Oxford Handbook of Jurisprudence and Philosopby of Law, Oxford: Oxford University Press, 125-165.

Hume, D., 1777: «Of the Standard of Taste» [1757], en D. Hume, Four Dissertations, London: A. Millar, http://www.davidhume.org/texts/fd.html.

KeEFe, R., 2008: «Vagueness: Supervaluationism», Philosophy Compass, 3: 315-324.

Kelsen, H., 1991: General Theory of Norms, transl. by M. HaRTnEy, Oxford: Oxford University Press.

LEWIS, D., 1978: «Truth in Fiction», American Philosophical Quarterly, 15: 37-48.

Luper, S., 2012: «The Epistemic Closure Principle», en E. N. ZalTA (ed.), The Stanford Encyclopedia of Philosophy (Fall 2012 Edition), URL: http://plato.stanford.edu/archives/fall2012/ entries/closure-epistemic/.

MacFarlane, J., 2014: Assessment Sensitivity. Relative Truth and its Applications, Oxford: Oxford University Press.

MaranhaO, J., 2013: Estudos sobre lógica e direito, Madrid-São Paulo: Marcial Pons.

Marmor, A., 2001: Positive Law and Objective Values, Oxford: Oxford University Press.

— 2002: «Exclusive Legal Positivism», en J. L. Coleman y S. J. ShaPIRO (eds.), The Oxford Handbook of Jurisprudence and Philosophy of Law, Oxford: Oxford University Press, 104-112.

- 2014: «Truth in Law», en A. MARMOR, The Language of Law, Oxford: Oxford University Press.

MendonCA, D., 1998: «Presumptions», Ratio Juris, 14: 399-412.

Montiel, J. P., y RAmírez, L., 2010: «De camareros estudiantes de biología a jueces biólogos. A propósito de la sentencia del BGH sobre los hongos alucinógenos y la deferencia a los expertos en el ámbito penal», en InDret, 1/2010, www.indret.com/code/getPdf. php? $i d=1344 \mathcal{E} p d f=691 . p d f \mathcal{E}$ indret.

MoOre, M., 1985: «A Natural Law Theory of Interpretation», Southern California Law Review, 58: 277-398.

Moreso, J. J., 1996: «On Relevance and Justification of Legal Decisions», Erkenntnis, 44: 73-100.

— 2010: «Tomates, hongos y significado jurídico», en J. J. Moreso, L. Prieto SANCHís y J. FERRER BELTRÁn, Los desacuerdos en el Derecho, Madrid: Fundación Coloquio Jurídico Europeo, 15-48.

- 2014: «Ethica more iuridico incorporata», en P. LuQue SÁNCHEZ y F. SCMARDELla (eds.), Gli argomenti del costituzionalismo. La tensione tra etica e diritto, Napoli: Editoriale Scientifica, 51-84. 
— 2015: «Positivismo jurídico contemporáneo», en J. L. FABRA ZAMORA y A. NÚÑEZ VAQUERO (eds.), Enciclopedia de Filosofía y Teoría del Derecho, vol. 1, México: UNAM, 171-205.

Moreso, J. J., y ChILOVI, S., ms.: «The Semantics and Pragmatics of According to the Law», on file con los autores.

Navarro, P. E., y Rodríguez, J. L., 2014: Deontic Logic and Normative Systems, Cambridge: Cambridge University Press.

NozicK, R., 1981: Philosophical Explanations, Cambridge, Mass.: Harvard University Press.

PerRY, J., 2011: «Textualism and the Discovery of Rights», en A. MARMOR y S. SoAmES (eds.), Philosophical Foundations of Language and Law, Oxford: Oxford University Press, 105-129.

PHILIPS, I., 2014: «Cetacean Semantics: A Reply to Sainsbury», Analysis, 74: 379-382.

RAz, J., 1980: The Concept of a Legal System, Oxford: Oxford University Press.

— 1985: «Authority, Law, and Morality», The Monist, 68: 295-324.

- 1994: Ethics in the Public Domain, Oxford: Oxford University Press.

- 2004: «Incorporation by Law», Legal Theory, 10: 1-17.

Rodríguez, J. L., 2002: Lógica de los sistemas jurídicos, Madrid: Centro de Estudios Políticos y Constitucionales.

Ross, A., 1957: «Tû-Tû», Scandinavian Studies in Law, 1: 137-153.

SAnsbury, M., 2014: «Fishy Business», Analysis, 74: 3-5.

Schauer, F., 2015: «Legal Fictions Revisited», en M. DEL Mar y W. TwINING (eds.), Legal Fiction in Theory and Practice, Dordrecht: Springer.

SchurZ, G., 1991: «Relevant Deduction. From Solving Paradoxes towards a General Theory», Erkenntnis, 35: 391-437.

SEARLE, J., 1995: The Construction of Social Reality, New York: The Free Press.

SHAPIRO, S. J., 2009: «Was Inclusive Legal Positivism Founded on a Mistake», Ratio Juris, 22: 326-338.

StALNAKeR, R., 2002: «Common Ground», Linguistics and Philosophy, 25: 701-721.

TOMÁs DE Aquino, 2000: Catena Aurea in Ioannem, Pamplona: Universidad de Navarra, http:// www.corpusthomisticum.org/.

Von Wright, G. H., 1963: Norm and Action. A Logical Enquiry, London: Routledge \& Kegan Paul.

Walton, K. L., 1990: Mimesis as Make-Believe, Cambridge: Cambridge University Press.

- 1994: «Morals in Fiction and Fictional Morality», Proceedings of the Aristotelian Society. Supplementary Volume, 68: 27-50. 